\title{
Changing the strategy and culture of stroke awareness education in China: implementing Stroke 1-2-0
}

\author{
Jing Zhao, ${ }^{1,2}$ Xutong Li, ${ }^{1}$ Xiaochuan Liu, ${ }^{3}$ Yuming $\mathrm{Xu},{ }^{4}$ Jihong $\mathrm{Xu},{ }^{5}$ Anding $\mathrm{Xu},{ }^{6}$ \\ Yongjun Wang, ${ }^{7,8}$ Renyu Liu (D) ${ }^{5}$
}

To cite: Zhao J, Li X, Liu X, et al. Changing the strategy and culture of stroke awareness education in China: implementing Stroke 1-2-0. Stroke \& Vascular Neurology 2020;5: e000324. doi:10.1136/ svn-2019-000324

- Additional material is published online only. To view please visit the journal online (http://dx.doi.org/10.1136/svn2019-000324).

Received 31 December 2019 Revised 15 February 2020 Accepted 2 March 2020 Published Online First 28 April 2020
Check for updates

(C) Author(s) (or their employer(s)) 2020. Re-use permitted under CC BY-NC. No commercial re-use. See rights and permissions. Published by BMJ.

For numbered affiliations see end of article.

Correspondence to

Dr Renyu Liu;

renyu.liu@pennmedicine.upenn. edu

\section{ABSTRACT}

This project implemented the Stroke1-2-0 stroke awareness programme across China and investigated its impact over a 2-year period. We initiated the Stroke1-2-0 educational campaign and Stroke1-2-0 special task forces (STF) across the nation. Massive media coverage, community-based educational sessions with videos and other related materials and induction of Stroke1-2-0 STF were the major means of promotion. We delivered a survey at the end of 2016 and 2018 to evaluate the impact of our effort. A total of 3066 participants responded to the first survey in 2016, and 15207 participants responded in 2018 across China. The acceptance rate for Stroke1-2-0 versus FAST (an English-language stroke awareness tool) was $50.2 \%$ versus $19.1 \%$ in 2016 , and changed significantly to $82.2 \%$ versus $8.0 \%$ in $2018(p<0.001)$. Stroke $1-2-0$ was well accepted by all ages and by people with different academic qualifications. Only $6.5 \%$ of survey respondents were aware that there was a therapeutic window for thrombolytic therapy in 2016, but this awareness increased significantly to $32.8 \%$ in 2018 . Only $12.6 \%$ of people in 2016 indicated that they would send patients with stroke to the nearest hospital capable of performing thrombolytic therapy, but there was a nearly threefold increase (52.5\%) in this number by 2018. More than 1000 major hospitals joined the Stroke1-2-0 STF, and more than 20000 'stroke warriors' have joined our stroke awareness improvement effort so far. Stroke1-2-0 stroke awareness programme is well-implemented and accepted, and is generating profound improvement in stroke awareness in China.

\section{BACKGROUND}

Stroke is still one of the leading causes of death worldwide, especially in developing countries. The mortality rate for stroke in China is five times higher than in the USA. ${ }^{1}$ Although intravenous thrombolytic therapy is available in China, a recent study indicated that only $1.6 \%$ to $4.0 \%$ of ischaemic stroke patients received such thrombolytic therapy, mainly due to significant prehospital delay, with a median time of prehospital delay of 15 hours in urban areas. ${ }^{12}$ The lack of effective programmes for stroke awareness is one of the main reasons for the high stroke mortality and disability rate in China. Very few stroke patients $(16.9 \%)$ could recognise the initial signs of stroke and less than $19 \%$ of them could make use of emergency medical services (EMS). ${ }^{3}$ Thus, there is an urgent need in China to educate people to improve stroke awareness and recognise that it is critical to use EMS immediately after the onset of a stroke. ${ }^{4}$

While FAST_face, arm, speech and time, a stroke awareness tool used in English-speaking countries based on the Cincinnati Prehospital Stroke Scale published in $1999^{5}$ —has been used in China for stroke awareness education for more than a decade, it did not work well due to the language barrier, and the prehospital delay is still devastatingly long. For this reason, we designed Stroke 1-2-0 as an education programme based on FAST and suitable for rapid stroke recognition and quick response in China. It uses 1-2-0, the well-known medical emergency phone number, as an easy mnemonic tool $(1$ represents 'First, look for an uneven face', 2 represents 'Second, examine for arm weakness' and 0 represents 'Zero (absence of) clear speech'), and link the quick recognition to the immediate activation of EMS by dialling 1-2-0. One of the most important strengths of Stroke 1-2-0 compared with FAST is that it eliminates the language barrier by using only the numbers that are used for EMS services. Since its publication in Lancet Neurology online on World Stroke Day in 2016, ${ }^{6}$ Stroke 1-2-0 was immediately adopted by the Chinese Stroke Association (CSA) as a unique tool for stroke awareness education, and was implemented into their stroke practice guidelines. $^{7}$ A Stroke 1-2-0 special task force (STF) was established, focussing on promoting stroke awareness across the nation. Here, we report how we implemented the novel strategy that profoundly changed stroke awareness education in China over a 2-year period.

\section{METHOD}

The Stroke 1-2-0 campaign

After the introduction of Stroke 1-2-0 on 29 October 2016, we initiated a nationwide 
Stroke 1-2-0 promotional campaign. With the support of the CSA we established the Stroke 1-2-0 STF focussing on promoting stroke awareness and started to establish local Stroke 1-2-0 STF across China. Along with this, 'Stroke 1-2-0 warriors' were recruited to join the STF. A dedicated website (www.stroke120.org) and a social media WeChat public platform (Chinastroke120, www.wechat.com) were also established to deliver the most updated information related to Stroke 1-2-0 STF activities. In order to make our strategy easier to be understood by all the people we also produced a Stroke 1-2-0 educational video which is only 1 min long. In this short video, we deliver the message of how critical it is to recognise stroke and trigger the medical emergency system as soon as possible. The video was translated into 22 different dialects to be understood across various regions in China, where the dominant languages are dialects, especially for older people. We created a series of posters, cartoons and lectures based on Stroke 1-2-0 for various educational scenarios for different audiences. Extensive publicity and promotion were achieved through massive media coverage and community-based educational sessions, as well as educational sessions in the schools across the country. We released the video to the public via multiple media platforms including Lancet Neurology (http://www. thelancet.com/journals/laneur/article/PIIS1474-4422( 16)30283-6/abstract, accessed on 13 October 2019), the Lancet Neurology Twitter account, China Central Television (CCTV) and local TV stations, as well as online video hosting sites that are popular in China such as Tencent video, Youku video and Ai Qi Yi video, as well as the official Stroke 1-2-0 website on 14 February $2017 .{ }^{8}$ In addition to the promotion of online media, Stroke 1-2-0 warriors across the country hosted educational sessions in hundreds of communities and schools to promote stroke awareness using Stroke 1-2-0 as a tool.

A

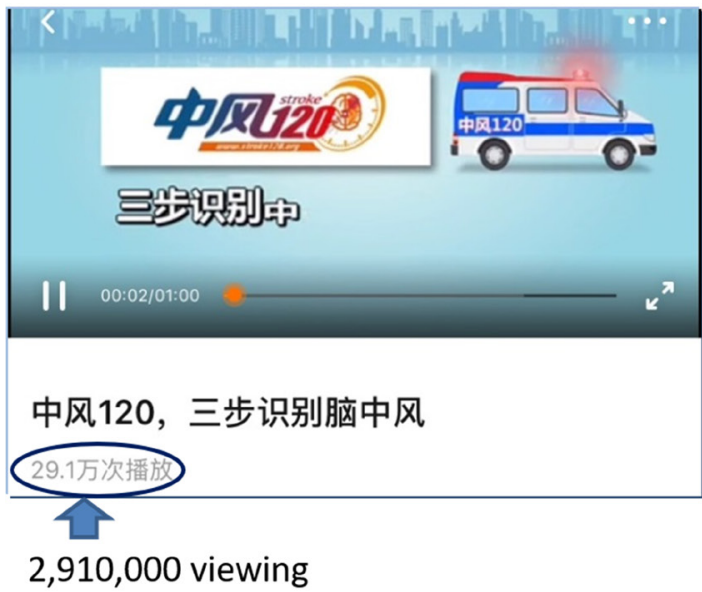

\section{Establishment of the Stroke 1-2-0 special task force}

The Stroke 1-2-0 special task force was first established by the CSA in February 2017 in Beijing, and led by JZ and $\mathrm{RL}$. The aim was to promote stroke awareness across the nation. More than 1000 major tertiary hospitals across China joined this effort within a 1-year period of time. By the end of 2018 the local Stroke1-2-0 special task forces had 16800 individual active members as the stroke warriors and were established in 12 provinces or special districts in China, covering 35\% of the areas in China.

\section{The campaign exposure}

The general acceptance of Stroke 1-2-0 was evident through its massive media coverage. As soon as Stroke 1-2-0 was published in Lancet Neurology online on the World Stroke Day, 29 October 2016, the CSA endorsed and promoted it on the same day in its official World Stroke Day event in Beijing, followed by over 200 regional and national news reports including a special introduction on China Central Television (CCTV) on 29 October 2016. Within hours of its broadcast on CCTV, its Weibo (a social media platform in China, similar to Twitter), had over 101 million views of its introduction with very positive reviews. The education video about Stroke 1-2-0 was released on 14 February 2017. The public accepted it very well. Many hospitals started to promote it through their broadcasting systems. Several TV stations broadcast it multiple times a day. Figure 1 shows two examples. One video had 5200000 views on one of the hosting sites, Tencent (Tengshun) (http://v.qq.com/), 1 month after its release. Many hospitals and television stations are broadcasting our Stroke 1-2-0 educational video repeatedly on a daily basis. An increasing trend for searching Stroke 1-2-0 was observed (figure 2). It is also important to note that Stroke 1-2-0 is now written into stroke care guidelines in China and it should be an essential component for stroke

\section{B}

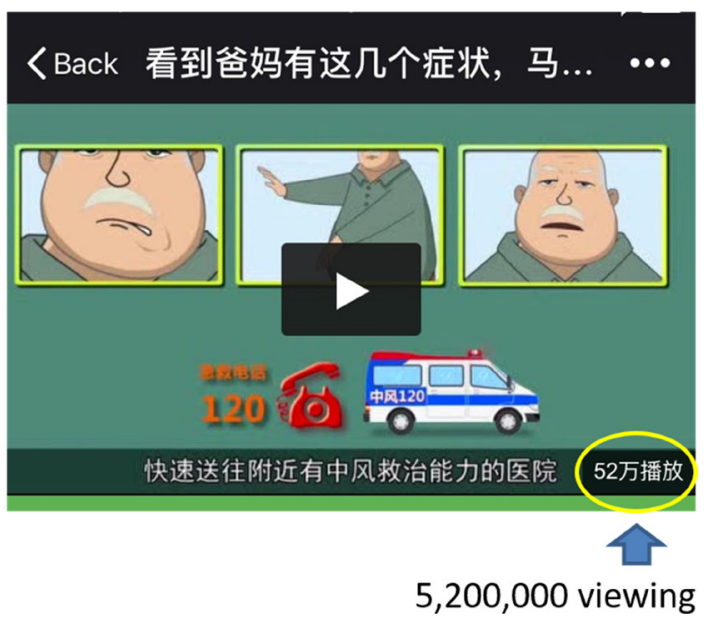

Figure 1 The educational videos for Stroke 1-2-0 have been watched over 200 million times on the websites during the first half year after their release. The left panel (A) indicates 2910000 viewings after 2 months of its release; the right panel (B) indicates 5200000 viewings on one of the hosting sites 1 month after its release. Such data indicate a general public interest spike on the awareness of stroke. 


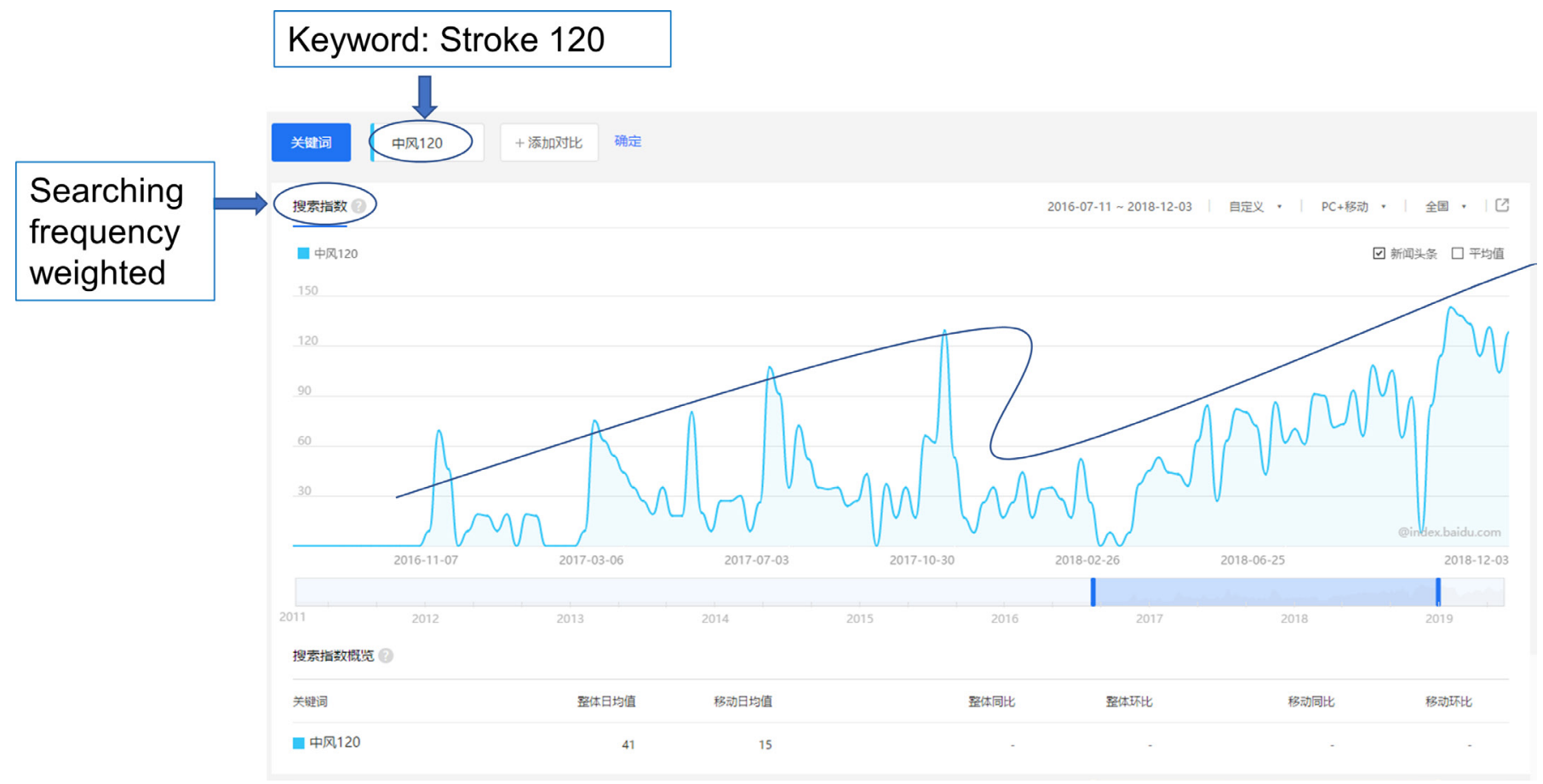

Figure 2 Searching weighted frequency of Stroke 1-2-0 extracted from Baidu Index from November 2016 to December 2018. As noted, there is no search of Stroke 1-2-0 in the early part of 2016; the search of Stroke 1-2-0 is gradually increasing over the period of 2016 to 2018 , indicating an increase in interest.

centre authentication. The National Health Commission of the People's Republic of China announced on 10 October 2019 that Stroke 1-2-0 should be used as a key educational tool for stroke awareness activities for the $14^{\text {th }}$ World Stroke Day (29 October 2019).

\section{Study design}

We designed an online survey using the professional online survey tool WJX.cn (https://www.wjx.cn). The survey included the following key questions: (1) What is your demographic information? (2) Which stroke educational tool is the easiest for you to remember? (3) Do you know the thrombolytic time window is 4.5 hours? (4) If someone around you had a stroke, what would you do? (5) What is your attitude toward informed consent for thrombolytic therapy? The details of the questions are listed in online supplementary appendix 1 . This survey was distributed to 30 provinces in China at two different time points, one in 2016 and one in 2018. It lasted only 1 month in December 2016, to collect baseline information without vigorous promotion, and 3 months in the fourth quarter of 2018. The primary end purpose was to determine the acceptance rate of Stroke 1-2-0 among the general public across the nation compared with FAST. That is, compared with FAST, Stroke 1-2-0 is more likely to be recognised by the Chinese, and the specific differences in their acceptance are analysed. The secondary purpose was improvement in stroke-related knowledge, specifically including "proportion of people who know the time window for thrombolytic therapy', 'proportion of response to stroke' and 'proportion of attitudes to informed consent for thrombolytic therapy'. From Baidu, the most popular online searching engine in China, we extracted the frequency-weighted figure of the key word 'Stroke 1-2-0' from the Baidu Index (http://index.baidu. $\mathrm{com} / \mathrm{v} 2 /$ index.html\#/) to indicate the public interest in our educational tool.

\section{Statistical analysis}

Categorical variables were expressed as either actual numbers or percentages using $\chi^{2}$ or Fisher's exact test. Statistical analysis was performed on SPSS V.20.0 (IBM Corp, Armonk, New York). Two-tailed $\mathrm{p}<0.05$ was considered statistically significant.

\section{RESULTS}

The characteristics of the responders in the survey

In 2016, a total of 3066 people from 30 different regions (provinces or cities) in China completed the survey. Major characteristics of the responders are presented in table 1 . Most of the survey responders $(89.5 \%)$ were between the ages of 21 to 60 years old, $11.0 \%$ of whom were neurologists (including physicians from neurology, interventional neurology and neurosurgery), and $8.2 \%$ were physicians who are not neurologists. City dwellers amounts to $87.8 \%$ of all responders. Two years later, in 2018, 15207 participants from 30 different regions (provinces or cities) took part in our online survey; $36.2 \%$ of them were male, $95.0 \%$ of them were at the age range of 21 to 60 years old, $16.3 \%$ of them were neurologists and $88.3 \%$ of all responders were city dwellers. 
Table 1 Characteristics of the responders in 2016 and 2018

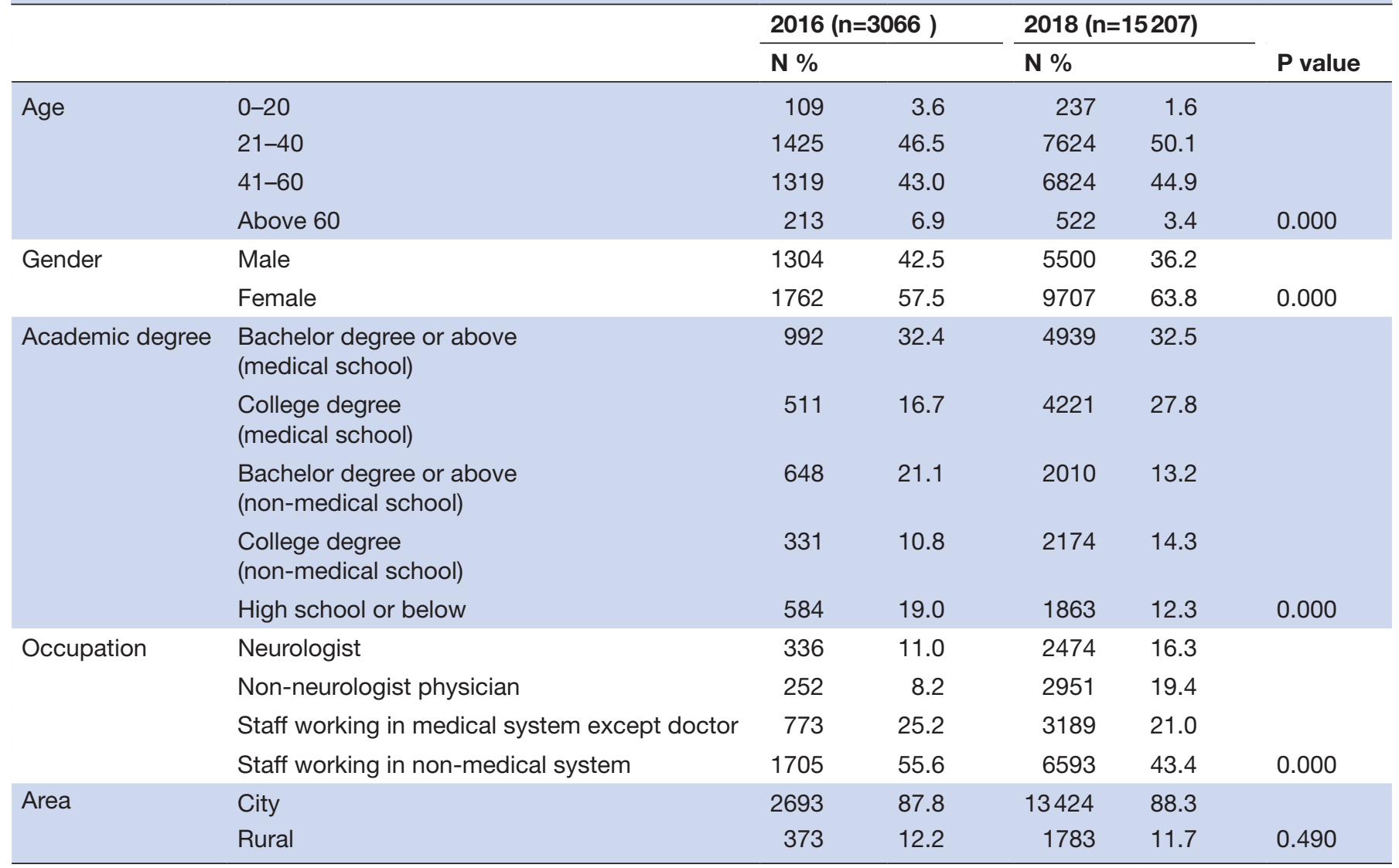

$\mathrm{n}$ is the total number of the responders of that specific year, $\mathrm{N}$ is the total number of the responders of the sub-variables in each main variable on the left column.

(1) please make sure \% align with the dedicated columns, both for 2016 and 2018, it should not together with N, they belong to different column, 2) please make sure there is space between " $n$ " and "is"

\section{The acceptance of Stroke 1-2-0}

According to the statistical results of Question 2, we considered that Stroke 1-2-0 is the easiest teaching tool to remember by calculating the proportion of the samples of the acceptance of Stroke 1-2-0 in the total samples (table 2). The acceptance rate for Stroke 1-2-0 was 50.2\%, compared with $19.1 \%$ for FAST among all the responders in $2016(\mathrm{p}<0.001)$. At the end of 2018, the acceptance rate of Stroke 1-2-0 increased to $82.8 \% \quad(p<0.001)$, with a significant decrease in FAST acceptance to $8 \%$ (figure 3A). Among neurologists, acceptance for Stroke $1-2-0$ versus FAST was $44.0 \%$ and $44.9 \%$, respectively, in 2016. Two years later, in 2018, the acceptance rate among neurologists for Stroke 1-2-0 increased dramatically to 95.9\%. Similar increases were seen in other occupation and age groups $(p<0.001)$. Stroke $1-2-0$ was well-accepted among people with different academic qualifications in both 2016 and 2018.

\section{The awareness of acute stroke management}

At the beginning of the Stroke 1-2-0 campaign, only $6.5 \%$ of the survey responders knew the therapeutic time window of intravenous recombinant tissue plasminogen activator; this number improved significantly to $32.8 \%$ after the campaign $(\mathrm{p}<0.001$; figure $3 \mathrm{~B})$. In
2016, only $12.6 \%$ of responders knew that it is critical to send someone who had a stroke to a nearby hospital with the capability of thrombolytic therapy, and after the campaign this number increased to $52.5 \%$ ( $\mathrm{p}<0.001$; figure 3C). A majority of the responders thought that an informed consent form should be signed before initiating thrombolytic therapy in $2016(63.8 \%)$ as well as in 2018 $(58.7 \%) .(p<0.001$; figure 3D).

\section{DISCUSSION}

This study clearly showed that Stroke 1-2-0 was well accepted across China. After 2 years of promotion, stroke awareness in China has improved significantly.

\section{From FAST to Stroke 1-2-0}

China has the largest number of stroke patients and the mortality and disability rate from stroke is much higher in China than that in well-developed countries. Unfortunately, the median prehospital delay in China for stroke victims is more than 15 hours, even in urban cities, mainly due to poor awareness. ${ }^{2}$ Thus, it is critical not only to establish a network of thrombolytic therapy stroke centres, but it is also critical to promote public stroke awareness. While various stroke identification tools 
Table 2 The comparison of the acceptance of FAST and Stroke 1-2-0 between 2016 and 2018

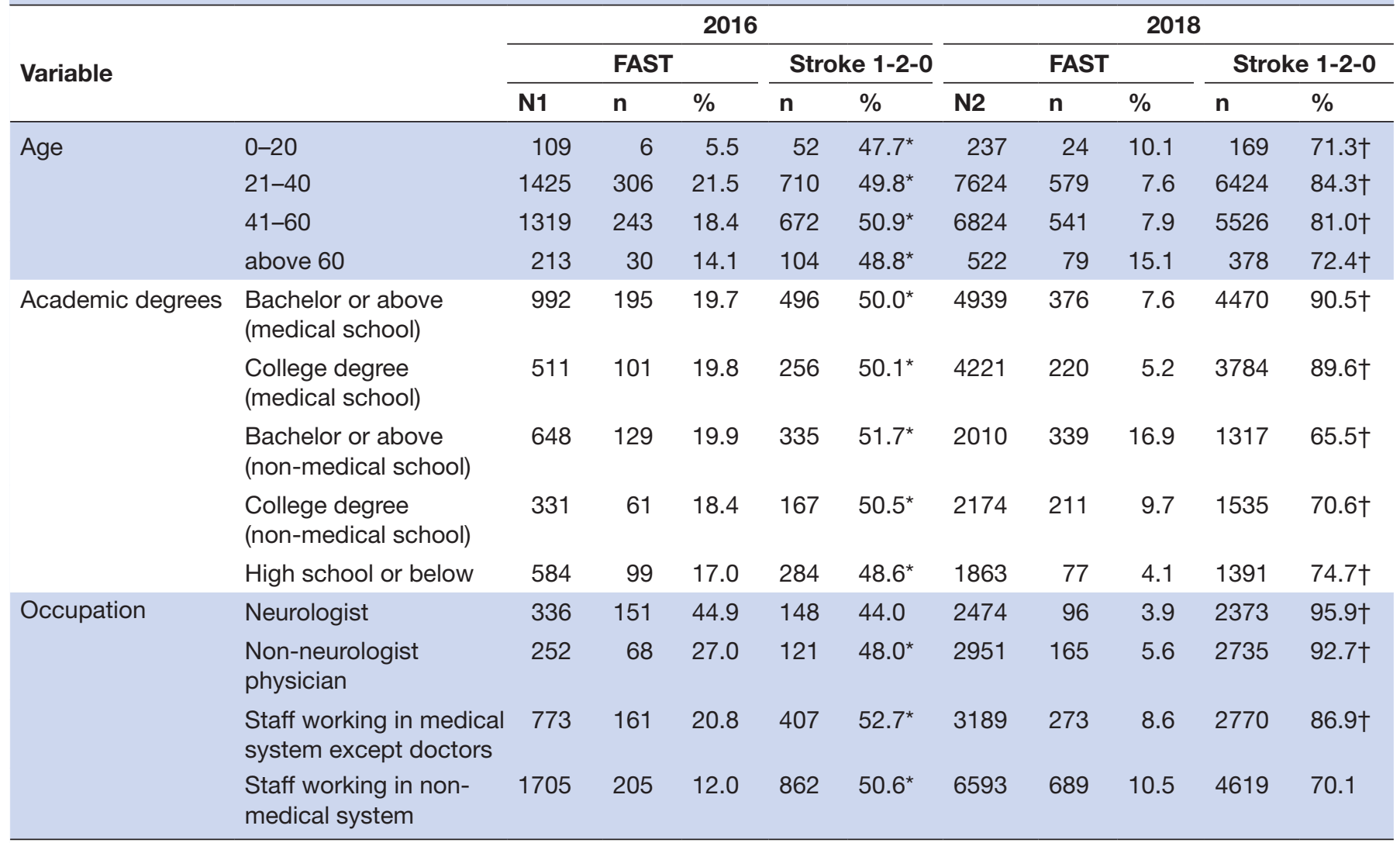

$\mathrm{N} 1$ represents the total number of responders for the main variables in 2016 . N2 represents the number of responders for the main variables in 2018. $\mathrm{n}$ represents the number of the responders for the defined sub-variables with the N1 or N2.

*Means $p<0.001$ between the acceptance rate of FAST and Stroke1-2-0 in 2016.

†Means $p<0.001$ between the acceptance rate of Stroke1-2-0 in 2016 and 2018.

have been developed, FAST was the most popular due to its simplicity and it was adopted in many countries and regions. FAST has been used in China as a stroke awareness education tool for over a decade. Its effectiveness, however, is doubtful in China, with its large population with various education levels and cultural backgrounds, as evidenced by the long prehospital delays and poor stroke awareness reported in the past. ${ }^{9}$ Stroke 1-2-0, a novel education programme based on FAST, eliminates the language barrier for the Chinese lay public. Through 2 years' promotion, the acceptance rate of Stroke 1-2-0 increased from 2016 to 2018, with a significant decrease in FAST acceptance. Stroke 1-2-0 was well-accepted by people in different age groups with different academic backgrounds. Most importantly, the acceptance rate of Stroke 1-2-0 among neurologists increased dramatically from 2016 to 2018, a major shift of concept. The acceptance of Stroke 1-2-0 was also evident by its massive media coverage. We believe that the acceptance of Stroke 1-2-0 will sustain its promotion over the years to come. Moreover, FAST will co-exist with Stroke 1-2-0 for quite a long time since English is the most popular second language in China and some of the experts still believe FAST is better than Stroke 1-2-0.

\section{The impact on stroke awareness}

A vast majority, $93.5 \%$, of the people who responded to the initial basic survey had never heard about the timesensitive thrombolytic therapeutic window for stroke treatment. Thus, it is critical not only to establish the network of thrombolytic therapy stroke centres, but also to improve awareness among the people to send stroke patients to the closest stroke centre or hospital where thrombolytic therapy is available. Despite improvement, the proportion of people who don't know the thrombolytic therapeutic treatment window of opportunity is still as high as $67 \%$. A majority of people do not realise the importance of stroke symptom identification and of sending a stroke patient to a nearby hospital immediately. As indicated in our survey results of 2016, only a small portion of the responders chose a nearby hospital that has the capability to perform thrombolytic therapy. After 2 years of promotion of Stroke 1-2-0, the proportion of responders choosing a nearby hospital with thrombolytic therapy available has risen significantly. Consent for thrombolytic therapy from the patient or family member is not needed in the USA, however an informed consent is needed in all hospitals in China. Informed consent by patient or family has been a significant hurdle in reducing the delay time to access time-sensitive thrombolytic 
A

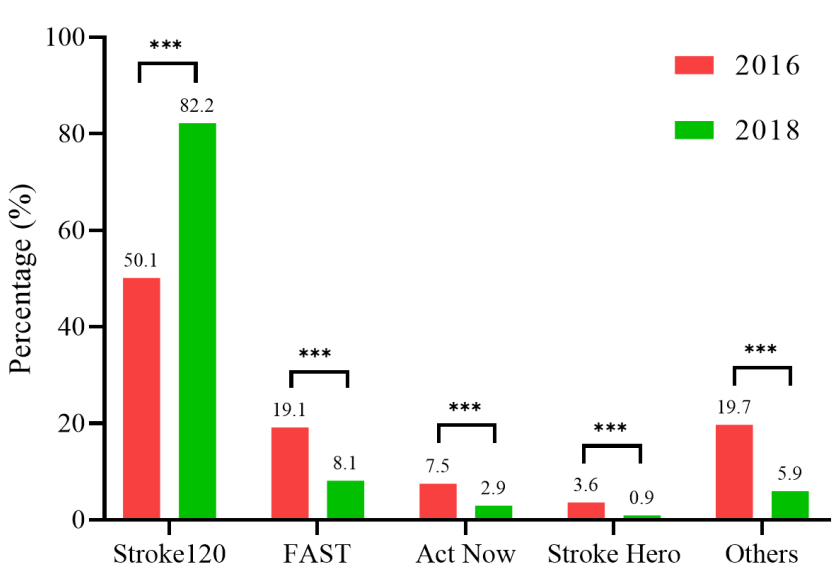

C

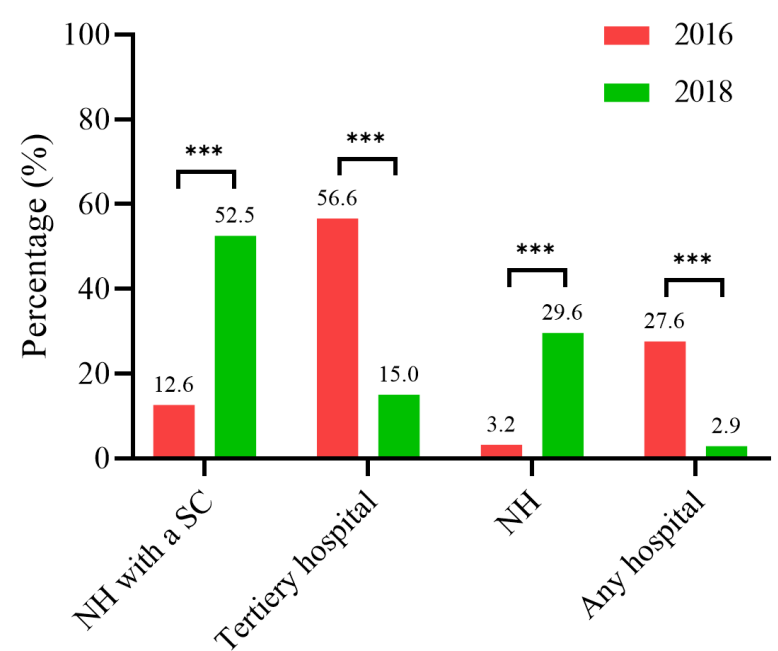

B
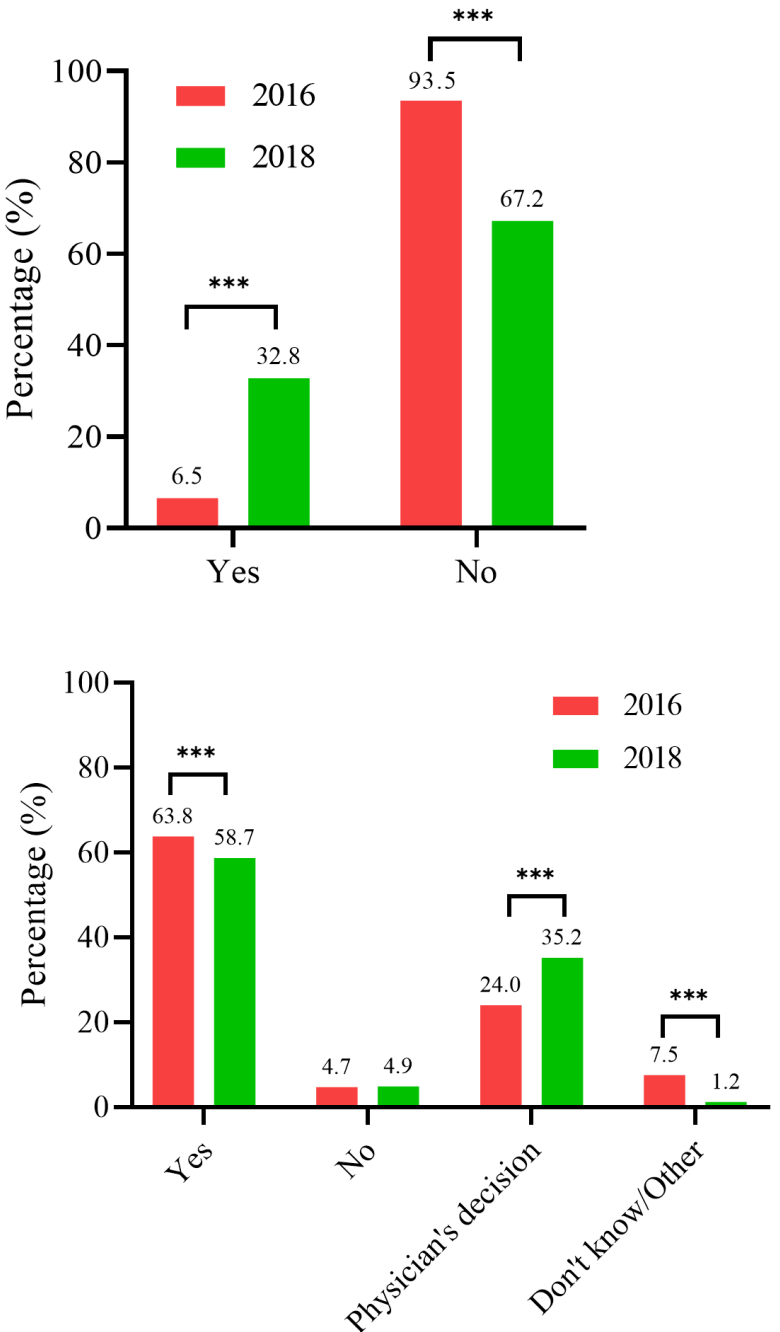

Figure 3 The impact of the Stroke1-2-0 campaign. (A) The acceptance rate of Stroke1-2-0 in 2016 and 2018; (B) Awareness of the thrombolytic time window for intravenous recombinant tissue plasminogen activator in 2016 and 2018; (C) How to choose a hospital after a stroke in 2016 and 2018? (D) Need for consent in 2016 and 2018. NH,nearby hospital; SC, stroke centreor facility with stroke care capability.

therapy. Despite our effort, the majority of the responders still believe that a consent is needed for thrombectomy. We believe that public education for stroke can shorten the time to obtain the necessary consent form. Elimination of the need for patient or family consent should be considered and promoted by the hospitals and by professional society leaders. ${ }^{9}$ This idea should be also supported by policy regulations to support neurologists and physicians who are eligible to perform thrombolytic therapy in a timely manner.

\section{The implications}

The implications of this study are enormous. With the availability of thrombolytic therapy and thrombectomy, prehospital delay has become the most critical limiting factor for rescue of stroke patients. Prehospital delay remains a major issue, especially in developing countries. Based on our recent review of available data and summary statement after an international symposium on stroke prehospital delay, we believe that the prehospital delay is a global health issue and a crisis needing immediate action. ${ }^{9}$ We believe that negative results of educational efforts may be caused by either using inappropriate tools or by non-vigorous implementation efforts. There is no shortcut to improve stroke awareness in the public domain. The use of a proper tool to improve stroke awareness is one of the most critical pathways to reduce prehospital delay globally.

\section{Limitations of the study}

The major limitation of this study is that it was an online survey type of investigation and we do not know how much of the potential audience that has been reached, we do not know what the response rate is and we do not know the opinion of these who received the survey but did not complete the survey. Moreover, in our survey population, the number of responders from urban residents are much larger than that from rural residents, so the representativeness of the survey population in the overall population may be affected. Subsequent studies 
focussing on rural areas may be needed. The main reason for this is that urban residents might be more concerned about medical knowledge and have higher enthusiasm for questionnaires. It is important to note that the two survey populations were not exactly matched, thus, the result of the comparison could potentially be biassed. Well-controlled studies will be needed in the future. Finally, it is unclear in this study whether the Stroke 1-2-0 has had an effect on the rate of thrombolysis treatment, or the mortality and disability rate after a stroke. Further studies need to be carried out on a much larger scale to demonstrate the effectiveness of this new education tool.

\section{CONCLUSIONS}

Stroke1-2-0 as a novel stroke awareness programme is wellimplemented and accepted, and is generating profound improvement in stroke awareness in various domains in China in a 2 year time frame. Consistent and vigorous education efforts using Stroke1-2-0 should continue in the future. We are actively collecting data to demonstrate whether our effort could reduce prehospital delay, increase thrombolytic utilisation rates and subsequently improve stroke mortality and morbidity.

\section{Author affiliations}

${ }^{1}$ Neurology, Minhang Hospital, Fudan University, Shanghai, China

${ }^{2}$ Department of Neurology, Fudan University, Shanghai, China

${ }^{3}$ Wannan Medical College, Wuhu, China

${ }^{4}$ Zhengzhou University First Affiliated Hospital, Zhengzhou, China

${ }^{5}$ Anesthesiology and Critical Care, University of Pennsylvania Perelman School of

Medicine, Philadelphia, Pennsylvania, USA

${ }^{6}$ Department of Neurology and Stroke Center, Jinan University First Affiliated Hospital, Guangzhou, China

${ }^{7}$ China National Clinical Research Center for Neurological Disease, Beijing, China

${ }^{8}$ Neurology, Tiantan Hospital Affiliated to Capital Medical University, Beijing, China

Contributors JZ and RL participated in designing the study, collecting data, analysing the data and writing the paper; the rest of the team participated in collecting data, analysing the data and writing the paper.
Funding We appreciate the following funding support from the National Natural Science Foundation of China (81572232, PI: JZ); Shanghai Natural Science Foundation (17dz2308400, PI: JZ); China Research Engagement Funding of the University of Pennsylvania (CREF-030, PI: RL).

Competing interests None declared.

Patient consent for publication Not required.

Ethics approval This study protocol was approved by the Institutional Review Board of Minhang Hospital, affiliated to Fudan University.

Provenance and peer review Not commissioned; externally peer reviewed.

Data availability statement Data are available upon reasonable request. Data are available upon reasonable request to Dr Jing Zhao, the first author of this paper.

Open access This is an open access article distributed in accordance with the Creative Commons Attribution Non Commercial (CC BY-NC 4.0) license, which permits others to distribute, remix, adapt, build upon this work non-commercially, and license their derivative works on different terms, provided the original work is properly cited, appropriate credit is given, any changes made indicated, and the use is non-commercial. See: http://creativecommons.org/licenses/by-nc/4.0/.

ORCID iD

Renyu Liu http://orcid.org/0000-0001-9335-8981

\section{REFERENCES}

1 Liu L, Wang D, Wong KSL, et al. Stroke and stroke care in China: huge burden, significant workload, and a national priority. Stroke 2011;42:3651-4

2 Jin H, Zhu S, Wei JW, et al. Factors associated with prehospital delays in the presentation of acute stroke in urban China. Stroke 2012;43:362-70.

3 Liu M, Wu B, Wang W-Z, et al. Stroke in China: epidemiology, prevention, and management strategies. Lancet Neurol 2007;6:456-64.

4 Zhao J, Liu R. Calling for a rapid recognition and response program for stroke in China. Trans/ Perioper Pain Med 2016;1:1-4.

5 Kothari RU, Pancioli A, Liu T, et al. Cincinnati prehospital stroke scale: reproducibility and validity. Ann Emerg Med 1999;33:373-8.

6 Zhao J, Liu R. Stroke 1-2-0: a rapid response programme for stroke in China. Lancet Neurol 2017;16:27-8.

7 Dong Q, Dong Y, Liu L, et al. The Chinese stroke association scientific statement: intravenous thrombolysis in acute ischaemic stroke. Stroke Vasc Neurol 2017;2:147-59.

8 Zhao J, Liu R. Stroke 1-2-0: the strategy and video release. Trans/ Perioper Pain Med 2017;2:1-2.

9 Zhao J, Ren L, Abraham S, et al. The stroke prehospital delay summary statement: a global battlefield. Trans/ Perioper \& Pain Med 2019;6:20-6. 